\title{
Solving Robust Glucose-Insulin Control by Dixon Resultant Computations
}

\author{
Laura Kovács \\ TU Vienna \\ Austria \\ lkovacs@complang.tuwien.ac.at
}

\author{
Béla Paláncz \\ Budapest University of \\ Technology and Economics \\ Hungary \\ palancz@epito.bme.hu
}

\author{
Levente Kovács \\ Budapest University of \\ Technology and Economics \\ Hungary \\ lkovacs@iit.bme.hu
}

\begin{abstract}
We present a symbolic approach towards solving the Bergman three-state minimal patient model of glucose metabolism. Our work first translates the Bergman threestate minimal patient model into the modified control algebraic Riccati equation. Next, the modified control algebraic Ricatti equation is reduced to a system of polynomial equations, and an optimal (minimal) solution of these polynomials is computed using Dixon resultants. We demonstrate the use of our method by reporting on three case studies over glucose metabolism.
\end{abstract}

Keywords-control engineering, glucose-insulin control, minimax optimization, polynomial equations, Dixon resultants

\section{INTRODUCTION}

According to the data provided by the World Health Organization (WHO), diabetes mellitus is predicted to be the "disease of the future" especially in the developing countries [1]. The diabetic population, representing $4 \%$ of the world's population in the year 2000 , is predicted to be doubled by the year 2030 and is being estimated to be $5,4 \%$ of the world's total population. The recent statistics of [2] show the same prevalence in terms of the period between years 2010-2030, and report on a significant increase in the overall diabetes population; for example $6.4 \%$ of the world's population in the year 2010 and an estimate of $7.7 \%$ in the year 2030.

Understanding and preventing the risks emphasized by these statistics requires a better understanding of the diabetes characteristics of a human body. It is well-known that the normal blood glucose concentration level in the human body varies in a narrow range, between $70-110 \mathrm{ml} / \mathrm{dL}$. If for some reasons the human body is unable to control the normal glucose-insulin interaction, for example the glucose concentration level is constantly out of the aforementioned range, diabetes mellitus, or simply just diabetes, is diagnosed. Depending on the blood glucose concentration level, diabetes is classified in various types, such as: (i) type I, also known as insulin dependent diabetes mellitus or IDDM; (ii) type II, also known as insulin independent diabetes mellitus or NIDDM; (iii) gestational diabetes (under pregnancy); and (iv) caused by genetic deflections. The consequences of diabetes are known to be mostly long-term; among others, diabetes increases the risk of cardiovascular diseases, neuropathy, and retinopathy [3].
Among the existing types of diabetes, we focus in this paper on type I diabetes mellitus. This type of diabetes is determined by the loss of insulin producing beta-cells, since they are completely destroyed by a still unknown autoimmune process [3]. Therefore, there is no human insulin production and an external insulin source has to be applied. For this reason, the question of artificial pancreas appeared and has been addressed throughout the past decades in order to enable the automatic control and treatment of diabetes mellitus [4], [5], [6].

The approach of a systematic artificial pancreas is to formulate the partially or totally deficient blood glucose control system of the human body as an engineering problem. To this end, from an engineering point of view, the treatment of diabetes mellitus can be represented by an outer control loop. As pointed out in [4], [5], the difficulty with engineering artificial pancreas comes with the design of (i) a continuous glucose sensor for measurements, (ii) an insulin pump for infusion, and (iii) a control algorithm. Moreover, an appropriate control algorithm crucially depends on the underlining computational model. For this reason, different mathematical models of the human blood glucose system have appeared during the last decades - see [6] for a brief overview.

The nonlinear model described in [7] proved to be the simplest one and was developed for type I diabetes patients under intensive care. However, its simplicity is also its disadvantage since in its formulation many components of the glucose-insulin interaction were neglected. Therefore, more general, but more complicated nonlinear models appeared, e.g. [8], [9], [10], [11], [12], where the nonlinear behavior of these models encode specific control aspects, such as stability, controllability, and observability. Consequently, control strategies for modeling the bloodglucose problem have been widely studied in the literature, e.g. [4], [5], [6]. Predictive or adaptive control proved to give adequate results if individualized treatment is desired. However, general requirements, like avoiding hypoglycemia (deviation under the basal glucose level) and minimizing hyperglycemia (deviation over the basal glucose level) are non-individual dependent "constraints", and these cannot be generally guaranteed by the aforecited control methods. 
Modern robust control methods are capable to handle such problems by designing optimal controllers on hard constraints [13], searching to determine the worst case of the system and determining an optimal solution for this case; hence, by designing a robust controller. Different methods are known in this topic, for example the $H_{2} / H_{\infty}$ method, also known as extended Linear Quadratic (LQ) method or minimax method; the pure $H_{\infty}$ method; or the $\mu$-synthesis method [13]. A detailed comparison and study of these methods in the modern robust control of the blood glucose system can be found in [14], [15].

However, all aforementioned methods are applicable efficiently only for linear models. Hence, to use these methods, the nonlinear models should be first linearized. Nonlinearity is however in general unavoidable in the proper mathematical modeling of artificial pancreas. Therefore, control methods developed directly for nonlinear systems are needed [16].

In [17] a differential geometric approach is proposed, combining advantages of linear-model based control theory, but it comes at the cost of high computational complexities of deployed numeric operations. The works described in [18], [19] overcome the difficulties of numeric computations and propose the use of formal methods, such as symbolic computation [20] and model checking [21], in the study of biological control systems. The control properties studied in [18] are expressed using temporal logic and describe changes between various states of the control model of a biological process. The formal analysis of these properties is then performed using symbolic model checking [21]. Characterizing the relation between the control states of a biological process is also addressed in [19]. However, unlike [18], the approach of [19] describes the nonlinear model as a collection of polynomial equations, and uses Gröbner basis computation [22] for solving the polynomial constraints. While the technique of [19] makes the first steps towards using symbolic computation in the artificial pancreas problem by deploying the Bergman-minimal model [7] and the $H_{2} / H_{\infty}$ method, it faces a number or practical challenges. To name a few, the polynomial constraints of the control model contains a large number of variables, and the initial states of the models are in general unknown.

In this paper we address these challenges and propose the use of Dixon resultants [23], for studying the diabetic system of glucose metabolism (Section IV). While glucose metabolism can be modeled in different ways, we make use of the Bergman three-state minimal patient model (Section II) for the following reasons. This model can be found in the background of any other model of glucose metabolism, and it can be used in conjunction with symbolic techniques for solving polynomial constraints, such as Gröbner basis computations or Dixon resultants. In our approach to solve the Bergman three-state minimal patient model, we first reduce the model to the modified control algebraic Riccati equation (MCARE) and then propose a method to symbolically solve the optimal solution of MCARE (Section III). Our solution uses the computation of Dixon resultant, and therefore benefits from the relatively small size of Dixon resultants (Section IV). Further, as Dixon resultants allow the elimination of more than one variable in one step of a computation, solving multivariate polynomial systems can become more efficient by using Dixon resultants. We demonstrate the use of our method by various case studies over glucose metabolism (Section V). All computations described in this paper were carried out using the symbolic computation framework of the computer algebra system Mathematica [24].

\section{PRELIMINARIES}

This section contains some definitions and properties about polynomials and matrixes (Section II-A), which are used in the rest of the paper. For additional details we refer to [25]. Next, we overview the Bergman three-state minimal patient control model of [7] (Section II-B), and present how this nonlinear control model can be linearized (Section II-C). Finally, the minimax method [13] is briefly introduced (Section II-D).

\section{A. Algebraic Considerations}

Throughout this paper we write $\mathbb{Q}$ and $\mathbb{C}$ to mean, respectively, the set of rational and complex numbers. For a complex number $z \in \mathbb{C}$, we denote by $\operatorname{Real}(z)$ and $\operatorname{Im}(z)$ its real part, respectively its imaginary part.

In the sequel, let $\mathbb{K}$ denote an arbitrary field of characteristic zero (e.g. $\mathbb{Q}, \mathbb{C}$, etc.). For variables $x_{1}, \ldots, x_{m}$, with $m \geq 1$, the ring $\mathbb{K}\left[x_{1}, \ldots, x_{m}\right]$ denotes the ring of polynomials in variables $x_{1}, \ldots, x_{m}$ with coefficients in $\mathbb{K}$. A polynomial $p\left(x_{1}, \ldots, x_{m}\right) \in \mathbb{K}\left[x_{1}, \ldots, x_{m}\right]$ is called symmetric if, after interchanging any of its variables, one obtains the same polynomial $p\left(x_{1}, \ldots, x_{m}\right)$. A polynomial equation is $p\left(x_{1}, \ldots, x_{m}\right)=0$. Throughout this paper whenever we refer to a solution or a root of $p\left(x_{1}, \ldots, x_{m}\right)$ we mean values of $x_{1}, \ldots, x_{m}$ such that $p\left(x_{1}, \ldots, x_{m}\right)=0$.

Given an $n \times m$ matrix $A$, we write by $A^{T}$ the $m \times n$ matrix denoting the transpose of $A$. $A$ is a square matrix if $n=m$. Further, a square matrix $A$ is called symmetric if $A=A^{T}$. Let $\bar{x}=\left(x_{1}, \ldots, x_{m}\right)$ denote a nonzero complex vector. An $m \times m$ square matrix $A$ with complex coefficients is a positive definite matrix if $\operatorname{Real}\left(\bar{x}^{T} A \bar{x}\right)>0$.

\section{B. The Bergman Three-State Minimal Patient Control Model}

In what follows, we use the following measures: $m g$ for milligrams, $m U$ for milliUnits, $\min$ for minutes and $d L$ for deciliters.

According to [7], the three-state minimal patient control model of glucose metabolism is characterized by the following three state variables (represented as functions over rationals with values in rationals): (i) $G(t)$ denoting the 
plasma glucose deviation measured in $m g / d L$, (ii) $X(t)$ representing the remote compartment insulin utilization measured in $1 / \min$, and (iii) $Y(t)$ denoting the plasma insulin deviation measured in $m U / d L$. The behavior of these state variables is characterized by the physical parameters as well as by the model parameters of the model. By physical parameters we mean the basal glucose level (denoted by $G B$ and measured in $m g / d L$ ), the basal insulin level (denoted by $Y B$ and measured in $m U / d L$ ), and the insulin distribution volume (denoted by $V L$ and measured in $d L$ ) in the model. In the sequel, the model parameters of the model will be denoted by $p_{1}, p_{2}, p_{3}$, and $p_{4}$, where $p_{1}, p_{2}, p_{4}$ represent measures in $1 / m i n$ and $p_{3}$ is $d L /\left(m U * m i n^{2}\right)$. The value of the physical and model parameters are given by [26]. However, to avoid round-off errors in representing these parameters, we consider the physical and model parameter to be rational constants. Following [26], we fix the values of the physical and model parameters as given below. To ease readability, we only list the values and omit their measures.

$$
\begin{array}{lll}
p_{1}=\frac{28}{10^{3}}, & p_{2}=\frac{25}{10^{3}}, \quad p_{3}=\frac{13}{10^{5}}, \quad p 4=\frac{5}{54}, \\
G B=110, & Y B=1.5, & V L=120 .
\end{array}
$$

We have now all ingredients to give the defining differential equations of the Bergman three-state minimal patient control model. These equations are listed below:

$$
\begin{aligned}
G^{\prime}(t) & =-p_{1} G(t)-X(t)(G(t)+G B)+h(t) \\
X^{\prime}(t) & =-p_{2} X(t)+p_{3} Y(t) \\
Y^{\prime}(t) & =-p_{4}(Y(t)+Y B)+i(t) / V L
\end{aligned}
$$

where $i(t)$ is the rational-valued control variable (input1) of the model representing the exogenous insulin infusion rate measured in $m U / \min$, and $h(t)$ is the rational-valued disturbance (input2) of the model representing the exogenous glucose infusion rate in $\mathrm{mg} /(d L *$ min $)$. We write $G^{\prime}(t)$ (respectively, $X^{\prime}(t)$ and $Y^{\prime}(t)$ ) to mean the partial derivative of $G(t)$ (respectively, of $X(t)$ and $Y(t)$ ) wrt $t$.

In general, it is assumed that the state variable $X(t)$ is a so-called slow variable ${ }^{1}$, that is $X^{\prime}[t]=0$ [19]. By making use of this assumption, the defining equations of (2) are reduced to the following model equations:

$$
\begin{aligned}
G^{\prime}(t) & =-p_{1} G(t)-\frac{p_{3}}{p_{2}} Y(t)(G(t)+G B)+h(t) \\
Y^{\prime}(t) & =-p 4(Y(t)+Y B)+i(t) / V L
\end{aligned}
$$

In what follows, we use (3) as the defining equations of the nonlinear diabetic control model under study.

\section{Linearized Control Model}

Note that (3) is a nonlinear differential equation system. Therefore, the first step in analyzing this model is to transform it into an equivalent, but linear system of differential equations. The linearization is done in the equilibrium state

\footnotetext{
${ }^{1}$ to be precise, $X^{\prime}(t)$ can safely be approximated by 0
}

of system (3) denoted by $X(0), Y(0), h(0)$, and $i(0)$, where $X(0)=0, Y(0)=0, h(0)=0$, and $i(0)=p_{4} * Y B * V L=$ 16.667. As a result, we derive the following linearized model of (3), written in matrix form:

$$
\begin{aligned}
x^{\prime}(t)= & \left(\begin{array}{cc}
-p_{1}-\frac{p_{3} Y(0)}{p_{2}} & -\frac{(G(0)+G B) p_{3}}{p_{2}} \\
0 & -p_{4}
\end{array}\right) x(t)+ \\
& \left(\begin{array}{cc}
1 & 0 \\
0 & \frac{1}{V L}
\end{array}\right) u(t) \\
y(t)= & \left(\begin{array}{ll}
1 & 0 \\
0 & 1
\end{array}\right) x(t)+\left(\begin{array}{ll}
0 & 0 \\
0 & 0
\end{array}\right) u(t),
\end{aligned}
$$

where $x(t)$ denotes the states of the system given by $(G(t)$, $Y(t)) ; y(t)$ represents the outputs of the system given by $(G(t), Y(t))$; and $u(t)$ denotes the inputs of the system given by $(i(t), h(t))$.

Following the notations of a linear time invariant statespace system [19], from (4) we get:

$$
\begin{aligned}
& x^{\prime}(t)=A x(t)+B u(t) \\
& y(t)=C x(t)+D u(t)
\end{aligned},
$$

where the following matrix equalities are satisfied: $A=$ $\left(\begin{array}{cc}-p_{1} & -\frac{G B p_{3}}{p_{2}} \\ 0 & -p 4\end{array}\right), B=\left(\begin{array}{cc}1 & 0 \\ 0 & \frac{1}{V L}\end{array}\right), C=\left(\begin{array}{cc}1 & 0 \\ 0 & 1\end{array}\right)$ and $D=\left(\begin{array}{cc}0 & 0 \\ 0 & 0\end{array}\right)$.

As a result, we thus obtained a linear system (5) representing the dynamics of the nonlinear system (3). By reducing (3) to (5), the nonlinear Bergman model has thus been linearized.

\section{The minimax control method}

In the study of the linearized Bergman three-state minimal patient model, after linearization, one is interested to find an optimal solution of (5). One way to find such an optimal solution is by deploying the classical linear quadratic (LQ) method [13], as follows. The LQ problem is defined to find an optimal solution of (5) by using the quadratic cost function:

$$
J(u(t))=\frac{1}{2} \int_{0}^{\infty} y^{T}(t) Q y(t)+u^{T}(t) R u(t) d t,
$$

where $R$ and $Q$ are unknown constant square matrices over the rationals, whose values effect the optimal solution and can be arbitrarily chosen. This represents the major disadvantage of the classical LQ method.

Therefore, the problem was extended by using modern robust control theory. The extension or the generalized version of the LQ method is called the minimax control method [13]. The starting point of the minimax method is to classify the the inputs of the system in two types, namely in wanted inputs (e.g. control inputs $u(t)$ ) and unwanted inputs (e.g. disturbances $h(t)$, appeared to perturb the system). Using this classification, the dynamics (5) of the linear 
model is changed by an explicit handling of the disturbance. That is, (5) is changed into the following system describing the dynamics of (4):

$$
\begin{aligned}
& x^{\prime}(t)=A x(t)+B u(t)+L h(t) \\
& y(t)=C x(t)+D u(t)
\end{aligned}
$$

where $L=\left(\begin{array}{ll}1 & 0 \\ 0 & 0\end{array}\right)$.

As a result, the quadratic cost function (6) of the LQ method is changed to the following cost function of the minimax method:

$$
J(u(t))=\frac{1}{2} \int_{0}^{\infty} y^{T}(t) y(t)+u^{T}(t) u(t)-\gamma^{2} h^{T}(t) h(t) d t,
$$

where $\gamma>0$ is a positive rational constant. Note that, unlike (6), in the case of using (8) we are left with only one unknown rational constant, that is with $\gamma$. Hence, the solution of $u(t)$ in (8) is parameterized only by $\gamma$.

To find the optimal control means to compute the value of $\gamma$, called the optimal value of $\gamma$, such that the value of (8) is minimised. The optimal value of $\gamma$ is computed by solving the below given modified control algebraic Riccati equation (MCARE) [13]:

$$
P A+A^{T} P+C^{T} C-P\left(B B^{T}-\frac{1}{\gamma^{2}} L L^{T}\right) P=0,
$$

where $P$ is a positive definite symmetric matrix, called the solution of MCARE. The challenge of finding $P$ comes however with the fact that, in the general case, $P$ is a complex matrix. The elements of $P$ are therefore not guaranteed to be rationals, although in the study of (7) and (8) we are interested to find rational solutions. Therefore, when solving (9) our goal is to find an optimal rational solution (see Section III) from which an optimum solution of $u(t)$ in (8) can be derived.

Note that in (8) the disturbance $h(t)$ appears with negative sign. Therefore, finding an optimum solution for $u(t)$ that minimizes the maximum cost of (8) is reduced to the problem of finding the worst case disturbance value of $h(t)$. Hence, a worst case design problem appears that can be written in the following minimax differential game:

$$
\max _{h(t)} J(u(t), h(t)) \rightarrow \min _{u(t)} J(u(t), h(t)) .
$$

According to [13], the differential game (10) admits a unique solution and its solution satisfies the following saddle-point condition:

$$
J\left(u^{*}(t), h(t)\right) \leq J(u(t), h(t)) \leq J\left(u(t), h^{*}(t)\right),
$$

where $u^{*}(t)$ is the optimal control solution for $u(t)$, and $h^{*}(t)$ denotes the worst-case disturbance. In addition, the optimal solutions for $u(t)$ and $h(t)$ satisfy the following equations:

$$
\begin{aligned}
& u^{*}(t)=-B^{T} P x(t) \\
& h^{*}(t)=\frac{1}{\gamma^{2}} L^{T} x(t)
\end{aligned}
$$

Minimizing the maximum cost of (8) requires thus computing the matrix $P$ such that (9) holds and $P$ is rational matrix. The challenge in deriving the optimal solutions (12) is therefore to compute the unknown rational elements of $P$. A standard approach for solving this challenge is to numerically approximate $P$. However, numeric computations can be expensive and crucially depend on the deployed precisions for representing rational/complex values. To overcome the burden of numeric representation, in what follows we propose a symbolic approach to compute the matrix $P$ as a rational matrix satisfying (9).

\section{Problem Setting and Statement: Solving MCARE}

Solving the linear model (7) of a diabetic control system comes with the task of deriving the optimal solution of the minimax differential game (10). To infer the optimal solution of (10) reduces however to compute the rational matrix solution of the MCARE system (9).

In what follows, let us focus our attention to solving (9), more precisely, to compute the unknown coefficients of the positive definite symmetric matrix $P$ satisfying (9). To this end, we consider $P$ as follows:

$$
P=\left(\begin{array}{ll}
p_{11} & p_{12} \\
p_{12} & p_{22}
\end{array}\right)
$$

where $p_{11}, p_{12}, p_{22}$ are the unknown constants whose values are to be determined. In the general case, $p_{11}, p_{12}, p_{22}$ are complex constants. However, in finding the optimal solution of (10) we are interested to derive $p_{11}, p_{12}, p_{22}$ as rational constants. That is, we seek solutions to the complex constants $p_{11}, p_{12}, p_{22}$ such that the imaginary parts of $p_{11}, p_{12}, p_{22}$ can be approximated by zero, and therefore minimized.

Substituting (13) in the left-hand-side of (9), the resulting matrix is symmetric. Further, using (13) together with the numeric values (1) of the physical and model parameters in (9), we obtain the following system of equations:

$$
\begin{cases}\frac{7 p_{11}}{125}+\frac{p_{12}^{2}}{14400}-\frac{p_{11}^{2}}{\gamma^{2}}-1 & =0 \\ \frac{p_{12}\left(\frac{26048}{15}+p_{22}\right)}{14400}-p_{11}\left(\frac{143}{250}-\frac{p_{12}}{\gamma^{2}}\right) & =0 \\ \frac{143 p_{12}}{125}+\left(\frac{5}{27}+\frac{p_{22}}{14400}\right) p_{22}-\frac{p_{12}^{2}}{\gamma^{2}}-1= & 0\end{cases}
$$

Note that the resulting system of equations (14) is parameterized by $\gamma$ and describes polynomial constraints over the unknowns $p_{11}, p_{12}$, and $p_{22}$. Let us denote by $\mathbb{T}$ the algebraic field $\mathbb{C}[\gamma]$. Then, (14) is a system of algebraic polynomials with variables $p_{11}, p_{12}$, and $p_{22}$, and with coefficients from $\mathbb{T}$. In other words, (14) describes polynomials from $\mathbb{T}\left[p_{11}, p_{12}, p_{22}\right]$. In what follows, for a fixed positive $\gamma \in \mathbb{Q}$, we respectively denote by $p_{11, \gamma}^{*}, p_{12, \gamma}^{*}$, and $p_{22, \gamma}^{*}$ the values of $p_{11}, p_{12}, p_{22}$ such that (14) is satisfied. 
Any solution of (14) determines the elements of $P$ in (9), and hence yields a solution of (10). However, in order to compute an optimal solution of (10), we need to ensure that the solution of (14) is minimal, in the following sense. Minimizing the solutions of (14) reduces to find the optimal $\gamma$, denoted by $\gamma_{\min }$, such that the imaginary parts of $p_{11, \gamma}^{*}, p_{12, \gamma}^{*}$, and $p_{22, \gamma}^{*}$ are minimal, that is (approximated to) zero. The optimal value $\gamma_{\min }$ of $\gamma$ thus ensures that $P$ is a rational-valued matrix. Current approaches for deriving $\gamma_{\text {min }}$ start from an arbitrarily chosen fixed $\gamma$ and iteratively approximate $\gamma_{\min }$ by computing the numerical solution of the multivariate polynomial (14) at every approximation step of $\gamma_{\min }$. Namely, an interval halving iteration process is computed to determine $\gamma_{\min }$. The halved intervals constrain the sought positive rational value for $\gamma$ and ensure a positive definite matrix $P$.

However, recent advances [19] suggest that, instead of minimizing the imaginary part of each element of $P$, it is sufficient to minimize the imaginary part of only one of the three unknowns of $P$. Therefore, in this paper, we propose to seek the minimal $\gamma_{\text {min }}$ such that the imaginary part of only one element of $P$, namely of $p_{12}$, is minimal. Hence, when solving (14) we also need to solve the following minimization problem:

$$
\gamma_{\min }=\min _{\gamma}\left(\operatorname{Im}\left(p_{12, \gamma}^{*}\right)\right)
$$

Unlike existing methods, in this paper we thus reduce the problem of minimizing the imaginary parts of solutions of a multivariate polynomial system to the problem of minimizing the imaginary part of the solution of a univariate polynomial system.

Summarizing, our goal in this paper is to derive an optimal solution of (10). For doing so, we need to solve (14) such that (15) is also satisfied. Using the notation above, we are therefore left with computing $\gamma_{\min }$ and $p_{11, \gamma_{\min }}^{*}, p_{12, \gamma_{\min }}^{*}$, and $p_{22, \gamma_{\min }}^{*}$. To this end, instead of using numeric approximations for minimizing (15), we propose to first compute $p_{12, \gamma}^{*}$ as a symbolic solution of (14) (see Section IV-B). For doing so, we deploy the computation of Dixon resultants (see Section IV-A). Next, $\gamma_{\min }$ is derived by making use of $p_{12, \gamma}^{*}$ and minimizing (15). We hence avoid solving numerically the multivariate polynomial system resulting from the minimization of (14), in every steps of the minimization process.

\section{MCARE REDUCTION TO DiXon RESUltANTS}

In Section III we showed that finding an optimal solution of (14) corresponding to the MCARE system reduces to minimizing (15). Minimizing (15) however comes with the task of solving a system of polynomial equations. We address this problem by deploying symbolic computation, as follows.

One of the classical methods of solving a system of polynomial equations is with so-called resultants. In our work, for solving and minimizing the polynomial system (15) constructed from the multivariate system (14), we make use of Dixon resultants (see Section IV-A). By using Dixon resultants, we then present our approach to derive optimal solutions of (14) (see Section IV-B). Moreover, as the Dixon resultants are relatively small in size, we argue that our method is computationally less expensive than other approaches using numeric techniques.

\section{A. The Dixon Resultant}

We begin by introducing the Dixon resultant for solving a system of polynomial equations [27], [28]. The Dixon resultant has various advantages over other resultant-based elimination methods for solving polynomial equations. To name a few, (i) the resulting matrix corresponding to the polynomial system has a considerably smaller size, hence, it is often easier to compute its determinant; (ii) a block of variables can be eliminated in one calculation, instead of using successive eliminations of variables. In the sequel, we overview the main ingredients of Dixon resultants.

Dixon resultants of univariate polynomials. We first recall Cayley's formulation [29] for solving a system of two polynomial equations. Consider two univariate polynomials $f(x), g(x) \in \mathbb{K}[x]$, and we denote by degree $(f)$, degree $(g)$ the degree of $f$ and $g$, respectively. Let $d e g=$ $\max ($ degree $(f)$, degree $(g))$ and let $a$ be a fresh new variable. Then,

$$
\begin{aligned}
\delta(x, a) & =\frac{1}{x-a} \operatorname{det}\left(\begin{array}{cc}
f(x) & g(x) \\
f(a) & g(a)
\end{array}\right) \\
& =\frac{f(x) g(a)-f(a) g(x)}{x-a}
\end{aligned}
$$

is a symmetric polynomial in variables $x$ and $a$ of degree deg-1. The polynomial $\delta(x, a) \in \mathbb{K}[x, a]$ is called the Dixon polynomial of $f$ and $g$. The common roots of $f(x)$ and $g(x)$ are also roots of $\delta(x, a)$ for every value of $a$. Consequently, at a common root, the coefficients of all powers $a^{i}$ in $\delta(x, a)$, with $i=0, \ldots, d e g-1$, are functions of $x$ and should be zero. Using matrix notation, we thus have the following homogeneous system in one variable:

$$
M\left(\begin{array}{c}
1 \\
x \\
\cdot \\
\cdot \\
x^{\operatorname{deg}-1}
\end{array}\right)=\left(\begin{array}{c}
0 \\
0 \\
\cdot \\
\cdot \\
\cdot \\
0
\end{array}\right),
$$

the columns of the $\operatorname{deg} \times \operatorname{deg}$ matrix $M$ consist of the coefficients of $a^{i}$. The matrix $M$ is called the Dixon matrix and its determinant $D=\operatorname{det}(M)$ is the Dixon resultant. System (17) has nontrivial solutions if and only if $D$ is zero. Therefore, $D=0$ is a necessary condition for the existence of common roots of $f$ and $g$.

Example 1: Let us consider the following example. We take $f(x)=x^{3}-2 x^{2}-11 x+12$ and $g(x)=x^{2}+3 x-4$, and 


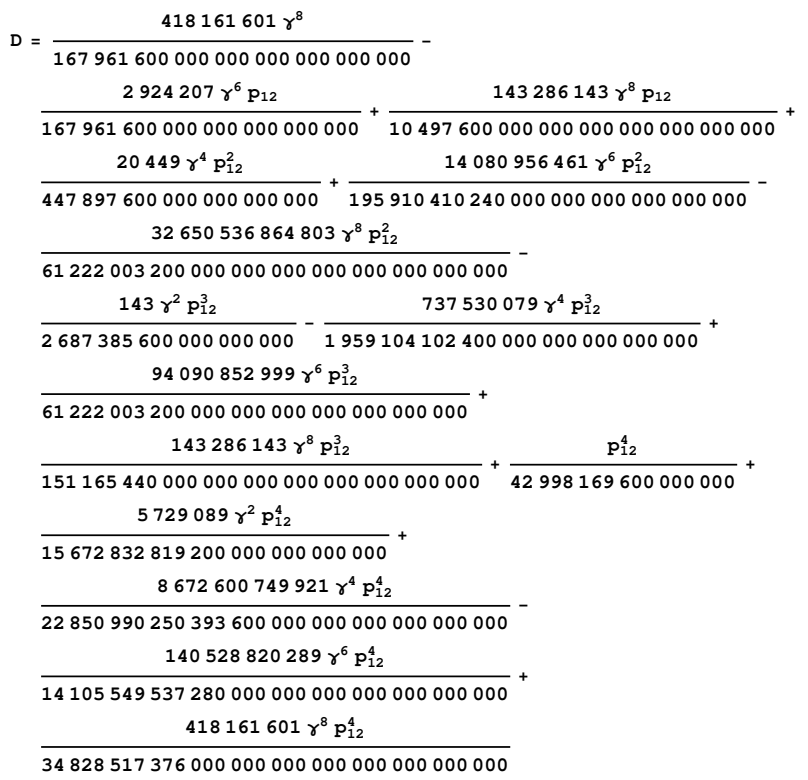

Figure 1. Dixon Resultant of MCARE (14), computed in Mathematica.

derive the Dixon polynomial $\delta(x, a)=8-4 a-4 a^{2}-4 x+$ $a x+3 a^{2} x-4 x^{2}+3 a x^{2}+a^{2} x^{2}$. By making the coefficient of powers of $a$ to be zero, (17) becomes:

$$
\left(\begin{array}{ccc}
8 & -4 & -4 \\
-4 & 1 & 3 \\
-4 & 3 & 1
\end{array}\right)\left(\begin{array}{c}
1 \\
x \\
x^{2}
\end{array}\right)=\left(\begin{array}{l}
0 \\
0 \\
0
\end{array}\right)
$$

The Dixon matrix is thus $M=\left(\begin{array}{ccc}8 & -4 & -4 \\ -4 & 1 & 3 \\ -4 & 3 & 1\end{array}\right)$ and its determinant $D$ is zero. Thus, $f(x)$ and $g(x)$ have common $\operatorname{root}(\mathrm{s})$, namely $x=1$.

Dixon resultants of multivariate polynomials. Cayley's formulation applies only to a system of univariate polynomials. Dixon has therefore generalized Cayley's approach to systems of multi-variate polynomials, more precisely, to a system of three polynomials with two variables, as follows. Take the polynomials $f(x, y), g(x, y), h(x, y) \in \mathbb{K}[x, y]$, and let $a, b$ be fresh new variables. The polynomial $\delta(x, y, a, b) \in$ $\mathbb{K}[x, y, a, b]$ is then defined as:

$$
\begin{aligned}
& \delta(x, y, a, b)= \\
& \frac{1}{(x-a)(y-b)} \operatorname{det}\left(\begin{array}{ccc}
f(x, y) & g(x, y) & h(x, y) \\
f(a, y) & g(a, y) & h(a, y) \\
f(a, b) & g(a, b) & h(a, b)
\end{array}\right)
\end{aligned}
$$

The common roots of $f(x, y), g(x, y)$ and $h(x, y)$ are also roots of $\delta(x, y, a, b)$ for every value of $a$ and $b$. Setting the power products $a^{i} b^{j}$ equal to zero, we again obtain a homogenous system in $x, y$. The coefficient matrix $M$ of this homogenous system is the Dixon matrix, and its determinant is the Dixon resultant $D$.
Similarly to the univariate case, Dixon proved that $D=0$ is a necessary condition for the existence of common roots of $f, g$, and $h$. Dixon's method easily generalize to a system of $n+1$ polynomials of degree $n$ with $n$ variables, where $n \geq 1$. However, Dixon resultants can also be used to solve a system of multivariate polynomials with $n+1$ variables of degree $n$ (or less). The idea is to consider the multivariate polynomials with $n+1$ variables and constant coefficients as multivariate polynomials with $n$ variables and coefficients parameterized by one variable of the original polynomial system. The example given below illustrates this idea.

Example 2: Let us consider the following example. Take $f(y, z)=x^{2}+y^{2}-1, g(y, z)=x^{2}+z^{2}-1$, and $h(y, z)=$ $y^{2}+z^{2}-1$. That is, $f, g, h \in \mathbb{T}[y, z]$, where $\mathbb{T}$ is $\mathbb{K}[x]$. In other words, the coefficients of $f, g, h$ are parameterized by $x$. We derive $\delta(y, z, a, b)=a b-2 a b x^{2}+b y-2 b x^{2} y+a z-$ $2 a x^{2} z+y z-2 x^{2} y z$ as the Dixon polynomial. By making the coefficient of powers of $a b$ to be zero, we obtain the Dixon matrix $M=\left(\begin{array}{cccc}0 & 0 & 0 & 1-2 x^{2} \\ 0 & 0 & 1-2 x^{2} & 0 \\ 0 & 1-2 x^{2} & 0 & 0 \\ 1-2 x^{2} & 0 & 0 & 0\end{array}\right)$.

The Dixon resultant is hence $D=1-8 x^{2}+24 x^{4}-$ $32 x^{6}+16 x^{8}$. To ensure that $f, g, h$ have common roots, we impose the condition that $D=0$. We are therefore left with solving a univariate polynomial equation in $x$. Any value of $x$ satisfying $D=0$, guarantees that $f, g, h$ have roots, and in addition, they have common roots. Note that substituting $x$ in $f, g, h$ by its value computed from $D=0$, the coefficients of $f, g, h$ parameterized by $x$ become constants. Hence, in solving the multi-variate polynomial system resulting from $f(y, z)=0, g(y, z)=0, h(y, z)=0$ one does not have to derive also the value of $x$.

As shown in Example 2, Dixon's generalized method can be applied to polynomials with symbolic coefficients (i.e. coefficients parameterized by variables). This property of Dixon's method allows the elimination of a block of variables by one calculation of the Dixon resultants. As the Dixon resultants are relatively small in size, we argue that solving a system of multivariate polynomials using Dixon resultants is a promising approach in various application domains, in particular, in the study of the diabetic system of glucose metabolism (Section IV-B).

\section{B. Solving MCARE by Dixon Resultant Computations}

Let us now reconsider the problem of finding an optimal solution of the MCARE system given in (14). Solving (14) means finding a common solution of the polynomial equations of (14). To this end, we propose to use the computation of Dixon resultants. Note however that (14) is a system of three polynomials with three variables and of degree two. Hence, when solving (14) we proceed in a similar manner as in Example 2, but also take into consideration the minimization problem of (15). That is, we are interested 


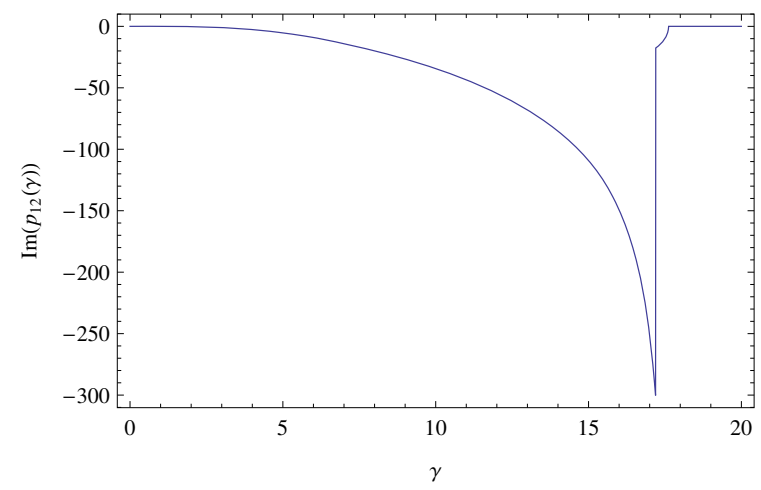

Figure 2. $p_{12}$ as function of $\gamma$, where $p_{12}$ satisfies $D=0$.

in solving (14), by also computing the value $\gamma$ for which the imaginary part of $p_{12}$ is minimized (and therefore, $p_{12} \in \mathbb{Q}$ ). To this end, we apply Dixon resultant computation such that we eliminate the variables $p_{11}, p_{22}$ and compute $p_{12}$ as a solution of the Dixon resultant. More precisely, we proceed as follows.

We consider (14) as a system of three 2-degree univariate polynomials in variables $p_{11}, p_{22}$, with coefficients parameterized by $p_{12}$. We next introduce the fresh new variables $a$ and $b$, and compute the Dixon polynomial $\delta\left(p_{11}, p_{22}, a, b\right)$ as given in (18). Setting further the power products $a^{i} b^{j}$ equal to zero, a homogenous system in $p_{12}$ is derived. The Dixon resultant $D$ computed for this univariate homogeneous system yields a 4-degree univariate polynomial in $p_{12}$, with coefficients parameterized by $\gamma$, as listed in Figure 1.

As $D=0$ is a necessary condition for the existence of a solution of (14), we are left with solving the univariate polynomial equation $D=0$. Any value of $p_{12}$ satisfying $D=0$ yields thus a solution of (14), however, not any value of $p_{12}$ gives an optimal solution of (10). In order to ensure that an optimal solution of (10) is computed, we need to solve the univariate polynomial equation $D=0$ such that (15) is also satisfied. That is, among the solutions of $D=0$ we compute $\gamma_{\min }$ such that the imaginary part of $p_{12}$ is minimized (i.e. it is zero). Hence, the solution $p_{12, \gamma}^{*}$ corresponding to $\gamma=\gamma_{\min }$ not only satisfies the polynomial equation $D=0$, but also yields an optimal solution of (10). To this end, by solving both $D=0$ and (15), we obtain:

$$
\gamma_{\min }=17.1968 \text {. }
$$

Figure 2 shows how the imaginary part of $p_{12}$ changes as function of $\gamma$, where $p_{12}$ is the (first) root of $D=0$.

Using that $\gamma_{\min }=17.1968$, we derive the value of $p_{12}$ and we replace $\gamma$ by $\gamma_{\min }$ in (14). This way, the numeric values of $p_{11}, p_{12}, p_{22}$ are obtained. As $D=0$ yields four roots, we obtain four different solutions for $p_{11}, p_{12}, p_{22}$. Out of the possible solutions for $p_{11}, p_{12}, p_{22}$, we choose the solution which makes matrix $P$ to be a positive definite matrix. We thus derive:

$$
P=\left(\begin{array}{cc}
299.512 & -2034.94 \\
-2034.94 & 14059.9
\end{array}\right)
$$

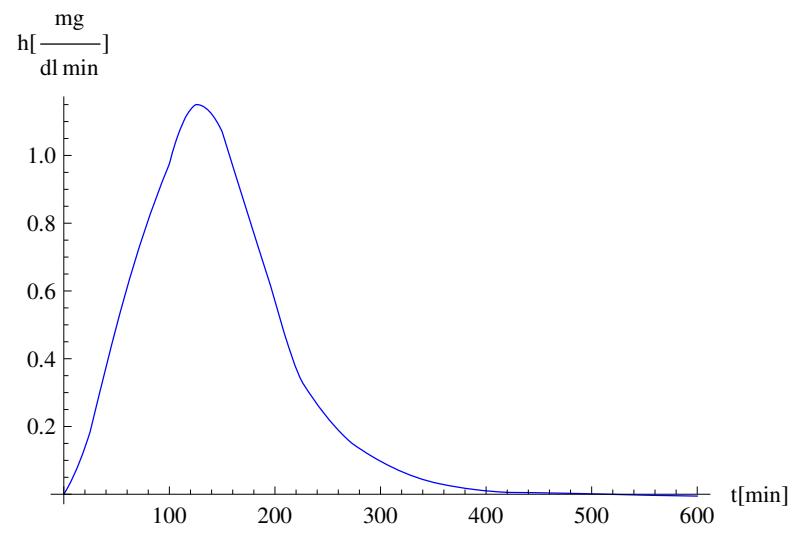

Figure 3. Exogenous glucose infusion $h(t)$ by meal.

as a solution of (9).

\section{Case Studies and Results}

We report on three case studies using Dixon resultants in the analysis of the presented blood-glucose problem (2). Our case studies were carried out with the computer algebra system Mathematica [24], by using the Mathematica package of [23] for computing Dixon resultants.

In our case studies we were interested on the efficiency and the robustness of our obtained control system solution, given by (19). In what follows, (19) will be regarded as our controller's MCARE solution. By replacing the solution (19) in the MCARE system (9), we derive the controller matrix $K L Q R$, called the gain matrix, as follows: $K L Q R=$ $\left(B^{T}+\frac{1}{\gamma_{\min }} L^{T}\right) P$. Substituting matrixes by their concrete values, we have:

$$
K L Q R=\left(\begin{array}{cc}
1.01279 & -6.88108 \\
-16.9579 & 117.166
\end{array}\right)
$$

In our case studies we used as meal input the classical six hour meal absorption profile modeled by [30] - see Figure 3. When testing the performance of our controller, our case studies reproduced the simulation scenarios used in [31].

Case study 1. In our first case study we turn back to the original nonlinear model (2), even though the design of KLQR was carried out on the reduced system (3). The glucose and insulin concentrations of (2) are shown in Figures 4 and 5. The results of our case study are in good agreement with the previous results of [31], [19], and they emphasize the use of our method. That is, using a methodology based on Dixon resultants and therefore different than state-of-the-art approaches, the same results can also be obtained. A more important question appears though when the robustness of our controller is investigated. The work of [31] shows that when limiting the model parameter $p_{1}$ to be zero, the system is unable to regulate the glucose level on its own. Indeed, when $p_{1}=0$, the eigenvalues of the linearized model are $(-0.0925926,-0.025,0)$, so the control system with $p_{1}=0$ is unstable. 

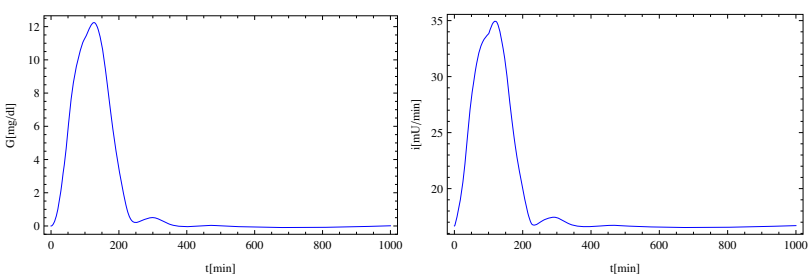

Figure 4. The controlled dynam- Figure 5. The insulin infusion ics of blood glucose concentration rate $i(t)$. $G(t)$

Case study 2. In our second case study the constraint $p_{1}=0$ was tested for the original nonlinear model (2). Our results showed that our controller is able to control (2) even in this case - see Figure 6. Moreover, the obtained results are better then [19] and thus demonstrate the use of Dixon resultants. However, it should be noted that the quality of the control when $p_{1}=0$ is not as precise as in the original case when $p_{1}=0.028$. This is due to the fact that, when $p_{1}=0$, a constant insulin infusion rate offset remains to compensate the system error even at the equilibrium state.

Case study 3. Our third case study focused on the robustness of the initial state of the nonlinear model (2). This question is crucial as identifying the right model parameters is hard due to, e.g., patient variability. Therefore, it is a nontrivial task to determine where the simulation of (2) should start from. Hence, a realistic sensor behavior performance is tested, as follows. The insulin infusion rate is considered to be nonnegative and its maximum value is limited. Figure 7 shows our results on simulating the nonlinear model (2) for a negative deviation of the initial values of the model. In the case of positive deviation of the initial values, we obtain similar simulation results. Our results thus show that the system performance can be stabilized fairly well even in the situations when the initial values of the model are deviated from their optimal values.

\section{CONCLUSIONS}

We present the use of Dixon resultants in the study of the Bergman three-state minimal patient model, in particular for computing optimal solution of the modified control algebraic Riccati equation of the model. Our approach benefits from the small size of Dixon resultants and from the elimination of block of variables using Dixon resultants. Moreover, the derived solutions are symbolic and we thus avoid the burden of successive numeric computations in inferring optimal solutions. We give practical evidence of our method by presenting three cases studies over glucose metabolism. Further work includes applying our approach to nonlinear models which are more complex than the Bergman threestate minimal patient model.

\section{ACKNOWLEDGMENT}

We acknowledge funding from the Hungarian-Austrian Aktion Foundation (project number 82öu11). Laura Kovács
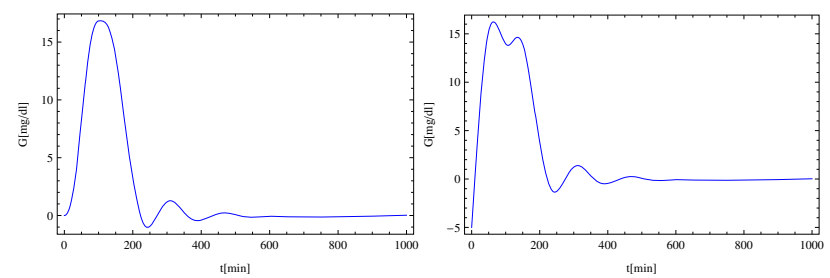

Figure 6. $G(t)$ in case of unstable Figure 7. $G(t)$ in case of but controllable system, where $p_{1}$ negatively shifted initial values is 0 ). $\quad$ and $p_{1}=0$.

is supported by the FWF Hertha Firnberg Research grant (T425-N23) and the FWF National Research Network RiSE (S11410-N23). Levente Kovács is supported by the János Bolyai Research Scholarship of the Hungarian Academy of Sciences. The work of Béla Paláncz and Levente Kovács is partially supported by the Hungarian National Scientific Research Foundation (grants number OTKA 82066 and CK80316). The research presented in this paper is partially funded by the scientific program of the "Development of quality-oriented and harmonized $\mathrm{R}+\mathrm{D}+\mathrm{I}$ strategy and functional model at BME" project, supported by the New Hungary Development Plan (project number TÁMOP-4.2.1/B09/1/KMR-2010-0002).

\section{REFERENCES}

[1] S. Wild, G. Roglic, A. Green, R. Sicree, and H. King, "Global Prevalence of Diabetes - Estimates for the Year 2000 and Projections for 2030," Diabetes Care, vol. 27, no. 5, pp. 10471053, 2004.

[2] J. E. Shaw, R. A. Sicree, and P. Z. Zimmet, "Global Estimates of the Prevalence of Diabetes for 2010 and 2030," Diabetes Research and Clinical Practice, vol. 87, pp. 4-14, 2010.

[3] A. Fonyó and E. Ligeti, Physiology (in Hungarian), 3rd ed. Medicina, Budapest, 2008.

[4] C. Cobelli, C. D. Man, G. Sparacino, L. Magni, G. de Nicolao, and B. Kovatchev, "Diabetes: Models, Signals, and Control (Methodological Review)," IEEE Reviews in Biomedical Engineering, vol. 2, pp. 54-96, 2009.

[5] R. Harvey, Y. Wang, B. Grossman, M. Percival, W. Bevier, D. Finan, H. Zisser, D. Seborg, L. Jovanovic, F. Doyle, and E. Dassau, "Quest for the Artificial Pancreas," IEEE Engineering in Medicine and Biology, vol. 29, no. 2, pp. 5362, 2010.

[6] F. Chee and F. Tyrone, Closed-loop Control of Blood Glucose, ser. LNCS. Springer-Verlag, Berlin, 2007, vol. 368.

[7] B. N. Bergman, Y. Z. Ider, C. R. Bowden, and C. Cobelli, "Quantitive Estimation of Insulin Sensitivity," American Journal of Physiology, vol. 236, pp. 667-677, 1979.

[8] C. D. Man, R. Rizza, and C. Cobelli, "Meal Simulation Model of the Glucose-Insulin System," IEEE Transactions on Biomedical Engineering, vol. 54, no. 10, pp. 1740-1749, 2007. 
[9] L. Magni, D. M. Raimondo, C. D. Man, G. D. Nicolao, B. Kovatchev, and C. Cobelli, "Model Predictive Control of Glucose Concentration in Type I Diabetic Patients: An In Silico Trial," Biomedical Signal Processing and Control, vol. 4, no. 4, pp. 338-346, 2009.

[10] R. Hovorka, V. Canonico, L. J. Chassin, U. Haueter, M. Massi-Benedetti, M. O. Federici, T. R. Pieber, H. C. Schaller, L. Schaupp, T. Vering, and M. Wilinska, "Nonlinear Model Predictive Control of Glucose Concentration in Subjects with Type 1 Diabetes," Physiological measurement, vol. 25, pp. 905-920, 2004.

[11] R. S. Parker, F. J. D. III, J. H. Ward, and N. A. Peppas, "Robust $H_{\infty}$ Glucose Control in Diabetes Using a Physiological Model,” AIChE Journal, vol. 46, no. 12, pp. 2537-2549, 2000.

[12] J. T. Sorensen, "A Physiologic Model of Glucose Metabolism in Man and its Use to Design and Assess Improved Insulin Therapies for Diabetes," Ph.D. dissertation, Dept. of Chemical Eng. Massachusetts Institute of Technology, Cambridge, 1985.

[13] K. Zhou, Robust and Optimal Control. Prentice Hall, New Jersey, 1996.

[14] L. Kovacs, "New principles and adequte control methods for insulin optimization in case of type i diabetes mellitus," $\mathrm{Ph} . \mathrm{D}$. dissertation, Budapest University of Technology and Economics, Hungary, 2007.

[15] L. Kovacs, B. Benyo, J. Bokor, and Z. Benyo, "Induced $\mathrm{L}_{2}$-norm minimization of glucose-insulin system for type $\mathrm{i}$ diabetic patients," Comp Meth Prog Biomed, Special issue on Glucose Modelling, vol. 102(2), pp. 105-118, 2011.

[16] A. Isidori, Nonlinear Control Systems, 3rd ed. Springer, Berlin, 1995.

[17] L. Kovács, P. Szalay, B. Benyó, and J. Chase, "Optimal Tight Glycaemic Control Supported by Differential Geometric Methods," in Proc. of 5th European Conference of the International Federation for Medical and Biological Engineering, 2011, pp. 351-354.

[18] H. Gong, P. Zuliani, and E. M. Clarke, "Model Checking of a Diabetes-Cancer Model," in Proc. of CMLS, 2011, pp. 234-243.

[19] L. Kovács and B. Paláncz, "Glucose-Insulin Control of Type 1 Diabetic Patients in $\mathrm{H}_{2} / \mathrm{H}_{\infty}$ Space Via Computer Algebra," in Proc. of AB, ser. LNCS, vol. 4545, 2007, pp. 95-109.
[20] B. Buchberger, "Symbolic computation: Computer algebra and logic," in Frontiers of Combining Systems, ser. Applied Logic Series. Kluwer Academic Publishers, 1996, vol. 3, pp. 193-219.

[21] E. M. Clarke, O. Grumberg, and D. A. Peled, Model Checking. MITPress, 1999.

[22] B. Buchberger, "Gröbner-Bases: An Algorithmic Method in Polynomial Ideal Theory," in Multidimensional Systems Theory - Progress, Directions and Open Problems in Multidimensional Systems, 1985, pp. 184-232.

[23] G. Nakos and R. Williams, "Elimination with the Dixon Resultant," Mathematica in Education and Research, vol. 6, no. 3, pp. 11-21, 1997.

[24] S. Wolfram, The Mathematica Book. Version 5.0. Wolfram Media, 2003.

[25] F. J. Ayres, Schaum's Outline of Theory and Problems of Matrices. New York: Schaum, 1962.

[26] S. M. Furler, E. W. Kraegen, R. H. Smallwood, and D. J. Chisolm, "Blood glucose control by intermittent loop closure in the basal mode: Computer simulation studies with a diabetic model," Diabetes Care, vol. 8, pp. 553-561, 1985.

[27] A. Dixon, "The Eliminant of Three Quantics in Two Independent Variables," London Mathematical Society, vol. 6, pp. 468-478, 1908 .

[28] A. D. Chtcherba, D. Kapur, and M. Minimair, "Cayley-Dixon Resultant Matrices of Multi-univariate Composed Polynomials," in Proc. of CASC, 2005, pp. 125-137.

[29] A. Cayley, "On the Theory of Elimination," Cambridge and Dublin Mathematical Journal, vol. III, pp. 210-270, 1865.

[30] E. D. Lehmann and T. A. Deutsch, "A physiological model of glucose-insulin interaction in Type1 diabetes mellitus," Journal of Biomedical Engineering, vol. 14, pp. 235-242, 1992.

[31] N. Hernjak and F. J. Doyle III, "Glucose control design using nonlinearity assessment techniques," AIChE Journal, vol. 51, no. 2, pp. 544-554, 2005. 